arthritis (RA) patients (pts). Personalised antidepressant treatment of ADD could be beneficial in managing of RA in this group of patients.

Objectives: To compare treatment response and remission rates in 4 groups of RA patients with ADD treated with DMARDs, biologics and antidepressants at the endpoint of a prospective 5 years study.

Methods: 128 RA-pts were enrolled in pilot study. All of them met the full ACR criteria for RA classification. $86 \%$ RA-pts were women with a mean age of $47,4 \pm 1,0$ $(\mathrm{M} \pm \mathrm{m})$ yrs. RA activity was evaluated with DAS28 and SDAI, remission was defined according to DAS28 $(\leq 2,6)$ and ACR/EULAR 2011 (SDAI $\leq 3,3$ ) remission criteria, response to treatment was classified according to EULAR and ACR/EULAR 2011 (SDAI) response criteria. Average DAS28 and SDAI scores at baseline were high and moderate $(5,25 \pm 0,16$ and $33,5 \pm 1,38(\mathrm{M} \pm \mathrm{m})) .62,6 \% \mathrm{RA}$-pts were taking prednisone $(9(5 ; 10) \mathrm{mg} /$ day (Me $(25 \% ; 75 \%)), 75,1 \%$ - DMARDs, $32 \%$ - biologics (rituximab - 12,5\%, anti-TNF- $\alpha-11 \%$, anti-IL6 - 6,2\%). ADD were diagnosed in $123(96,1 \%)$ of RA-pts by psychiatrist in accordance with ICD-10 in semi-structured interview. Severity of anxiety and depression was evaluated with Montgomery-Asberg Depression Rating Scale (MADRS) and Hamilton Anxiety Rating Scale (HAMA). RA-pts with ADD were divided into the following treatment groups: 1 - DMARDS ( $n=39), 2$ - DMARDs+antidepressants (sertraline or mianserine) ( $n=43$ ), 3 - DMARDs+biologics ( $n=32), 4$ - DMARDs+biologics+ antidepressants (sertraline or mianserine) $(n=9)$. Biologics treatment duration varied from 1 to 6 years, antidepressants - from 6 to 96 weeks. Differences in baseline disease activity scores were significant only in DAS28 for group 1 versus group 4 (4,95 $\pm 0,24$ vs $6,45 \pm 0,52 ; p=0,026$ ).

Results: The RA-treatment efficacy was evaluated at 5 years endpoint in 83 RApts. The percentage of patients who achieved good response according to EULAR (DAS28) criteria was higher $(p<0,05)$ in groups $2(41,4 \%)$ and $3(28,6 \%)$ vs DMARDs (4,2\%) group. In biologics groups (3 and 4 ) good response according to SDAI criteria was more $(p<0,05)$ prevalent $(52,4 \%$ and $88,8 \%$ respectively) than in group $2(25 \%)$, and vice versa for moderate response. In addition, patients in biologics groups achieved good response significantly more often than moderate $(p<0,05)$. EULAR and SDAI nonresponse rates were significantly lower in $2-4$ vs DMARDs groups. Patients treated with DMARDs+antidepressants achieved remission significantly more often $(p=0,024)$ than ones receiving DMARDs only. Remission by ACR/EULAR 2011 criteria was reached exclusively by DMARDs+antiidepressants patients.

Conclusions: our findings demonstrate that successful treatment of ADD with antidepressants provides more significant positive influence on treatment response to DMARDs and biologics in rheumatoid arthritis patients on a five-year follow-up. Diagnosis and treatment of ADD would potentially play an important role in individualised management of RA patients

Disclosure of Interest: None declared

DOI: 10.1136/annrheumdis-2018-eular.1430

\section{THU0148 $\quad$ SCREENING SYSTEM FOR EARLY ARTHRITIS WITH HEALTH PROFESSIONAL ASSISTANTS - A PROJECT OF THE T2T INITIATIVE IN GERMANY}

C. Jacobsen ${ }^{1}$, V. Höhne-Zimmer ${ }^{1}$, T. Braun¹, V. Köhler ${ }^{1}$, T. Leipold ${ }^{2}$, B. Tenckhoff ${ }^{2}$, K. Karberg ${ }^{3}$, G.R. Burmester ${ }^{1}$, J. Detert'. ' ${ }^{1}$ Department of Rheumatology and Clinical Immunology, Charité - Universitätsmedizin Berlin; ${ }^{2}$ Clinpath $\mathrm{GmbH} ;{ }^{3}$ Rheumapraxis Steglitz, Berlin, Germany

Background: Early stages of rheumatic diseases are still difficult to diagnose and treatment is delayed often due to the lack of practicing rheumatologists. Therefore, novel ways of diagnostic strategies are urgently needed.

Objectives: Evaluation of a structured screening system for selecting and treating patients (pts) with rheumatoid arthritis (RA) or rheumatic and musculoskeletal diseases (RMD) with health professional assistants (HPA, trained specialist nurses).

Methods: 177 pts visited a screening appointment for early arthritis (EA) between February 2015 and July 2016 in a specialised EA clinic. Inclusion criterion was arthritis in $\geq$ one joint for less than one year. Pts had three options for accessing the screening: phone call with qualified HPAs, online questionnaire or attending a walk in clinic (figure 1). Upon screening, all pts filled in a digital questionnaire about their symptoms and comorbidities. In group 1 an HPA performed the joint count and analysed the questionnaire for 116 pts before giving a suspected diagnosis. Subsequently, a rheumatologist saw these pts and also made a suspected diagnosis. 61 pts in group 2 were examined directly by a rheumatologist. In case of a suspected RMD or abnormal laboratory parameters, pts received a new appointment for completing diagnostics, or in acute cases, treatment was started immediately. All pts had the opportunity of a new appointment in case of persistency or worsening of the symptoms.

Results: Pts had a mean age of $50.9 \pm 15.2$ years and 135 (76.3\%) pts were female. $160(90.4 \%)$ pts had access to the screening by phone call. $10(5.7 \%)$ pts used the online questionnaire, and $7(3.9 \%)$ pts used the walk-in consultation. Pts waited $3.1 \pm 1.8$ weeks for a screening appointment. According to the digital questionnaire pts had symptoms for $58.1 \pm 90.5$ weeks at the screening appointment.
$34(56.7 \%)$ pts with RMD visited the screening clinic within six months after symptom onset. $2(1.7 \%)$ pts had an RMD that had not been suspected by the HPA upon screening in group 1 and subsequently received conventional disease modifying antirheumatic drugs (cDMARDs) and glucocorticoids (GC). In group 2, 3 $(4.9 \%)$ pts received cDMARDs although in the screening an RMD had not been initially suspected by the rheumatologist. In total $69(39.0 \%)$ pts finally had an RMD, whereof $43(24.3 \%)$ pts had an RA. 44 (24.9\%) pts received therapy with a cDMARDs and $6(3.4 \%)$ had the recommendation for a therapy with cDMARDs but refused treatment. Therapy with cDMARDs started $44.8 \pm 41.9$ days after screening. $21(11.9 \%)$ pts could already start with GC at the screening appointment. $27(15.2 \%)$ pts without a diagnosed RMD visited the rheumatologist at least twice.

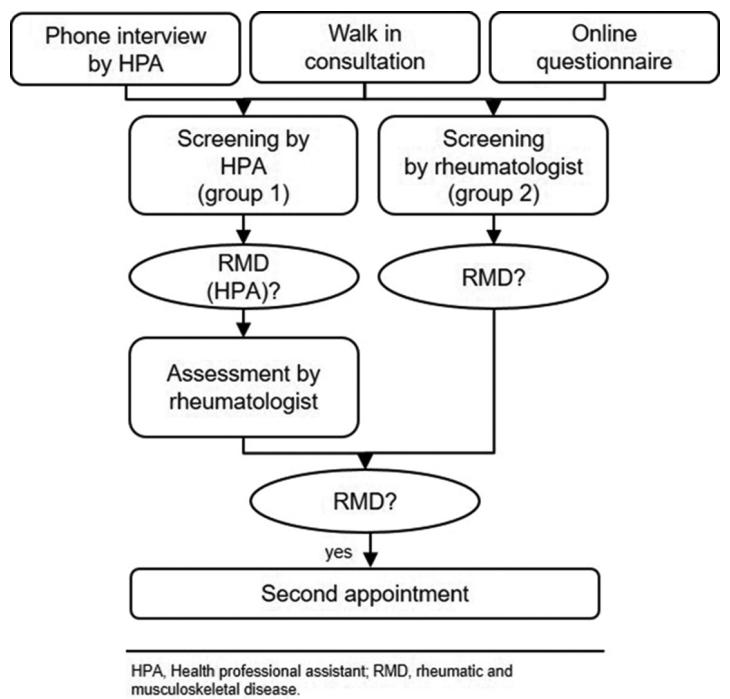

Abstract THU0148 - Figure 1. HPA, Health professional assistant; RMD, rheumatic and musculoskeletal disease.

Conclusions: HPAs can select pts with RMDs efficiently in a structured screening system which leads to treating RMDs at an early stage in times of limited resources.

Acknowledgements: Abbvie supported the project within the T2T Initiative Germany.

Disclosure of Interest: None declared

DOI: 10.1136/annrheumdis-2018-eular.6969

\section{THU0149 TRENDS IN THE INCIDENCE OF INFECTION IN PATIENTS WITH RHEUMATOID ARTHRITIS IN SPAIN: AN OBSERVATIONAL COHORT STUDY OF HOSPITAL DISCHARGES FROM 1999 TO 2015 (TREND-AR STUDY)}

C. Martinez-Prada ${ }^{1}$, R. Mazzuccheli ${ }^{2}$, I. Morado $^{1}$, E. Perez-Fernández ${ }^{2}$, J. Quirós ${ }^{2}$, J.L. Morell-Hita ${ }^{3}$, M. Peña ${ }^{3}$, N. Crespi ${ }^{4}$, C. Barbadillo ${ }^{5}$, H. Godoy ${ }^{5}$,

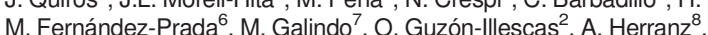
V. Villaverde ${ }^{9}$, A. García-Vadillo ${ }^{10}$. ${ }^{1}$ Hospital Clínico San Carlos; ${ }^{2}$ Hospital Universitario Fundación Alcorcón; ${ }^{3}$ Hospital Univrsitario Ramón y Cajal; ${ }^{4}$ Centro de Salud La Rivota; ${ }^{5}$ Hospital Universitario Puerta de Hierro; ${ }^{6}$ Hospital Universitario Guadalajara; ${ }^{7}$ Hospital Universitario Doce de Octubre; ${ }^{8}$ Hospital del Henares; ${ }^{9}$ Hospital Universitario de Móstoles; ${ }^{10}$ Hospital Universitario La Princesa, Madrid, Spain

Background: Rheumatoid Arthritis (RA) patients are at an increased risk of infection compared with healthy individuals, related to immune dysfunction. New treatments have revolutionised RA management; however, serious infection especially in elderly remains a concern.

Objectives: To analyse the incidence and trend of hospital admissions for all infections in patients with RA in Spain during the period between 1999 and 2015. Methods: This is a national retrospective population based study. We analysed a national administrative database that includes a Minimum Basic Data Set (MBDS) in all hospital admissions of patients with RA. Period: 1999 to 2015. Cases were identified by the presence of ICD9 codes. The population at risk was estimated through the population census of the National Institute of Statistics. The adjusted rates of infection were calculated, by sex and age. The trend was analysed by Generalised Linear Models (GLM). Statistical analysis was made using SPSS statistical package version 20 (SPSS Inc, Chicago, IL). 